\title{
Adherencia terapéutica de pacientes hipertensos de la comunidad de Honorato Vásquez.
}

Therapeutic adherence of hypertensive patients in the community of Honorato Vásquez.

Luisa Katerine Bustamante Espinoza. ${ }^{1}$, Mayra Teresa Cruz Gavilanez. ${ }^{2} \&$ Maria Erlinda Aguaiza Pichazaca. ${ }^{3}$

Recibido: 17-03-2017 / Revisado: 09-05-2017 Aceptado: 04-06-2018/ Publicado: 01-07-2018

\begin{abstract}
.
DOI: https://doi.org/10.33262/cienciadigital.v2i3.180

The general objective is to describe and analyze the therapeutic adherence of hypertensive patients of the Honorato Vasquez community. A descriptive, crosssectional, quantitative study of 50 patients diagnosed with arterial hypertension residing in the Honorato Vásquez province of Cañar province was carried out. April 2018 to May 2018 and determine the therapeutic adherence of hypertensive patients, where there is a partial way to carry out the treatment is common, having a low appreciation about the complications that this pathology entails, the people who participated in the surveys had difficulties to comply with the treatment prescribed by the specialist, one of which is the awareness that would be the main barrier to compliance with it, downplaying the impact on your health of taking the treatment under the guidelines indicated, so you can see affected the fidelity of it either in the far way macological and its measures in close relation with lifestylesm.
\end{abstract}

Keywords: Arterial Hypertension, Adherence, Therapeutic.

\section{Resumen.}

El objetivo general es describir y analizar la adherencia terapeutica de pacientes hipertensos de la comunidad de Honorato Vasquez, se cumplió con un estudio.

1 Universidad Católica de Cuenca, Hospital Luis F Martínez, Cuenca Ecuador., kbustamantee@ucacue.edu.ec

2 Universidad Católica de Cuenca, Hospital Homero Castanier Crespo, Cuenca Ecuador, mtcruzg@ucacue.edu.ec

3 Universidad Católica de Cuenca, Cuenca, Ecuador, eaguaizap@ucacue.edu.ec 
cuantitativo descriptivo, transversal de 50 pacientes diagnosticados de hipertensión arterial, que residen en la comunidad de Honorato Vásquez provincia del Cañar, desde abril 2018 a mayo de 2018 y determinar adherencia terapéutica de los pacientes hipertensos, donde que existe una forma parcial de llevar el tratamiento es común, teniendo una baja apreciación sobre las complicaciones que conlleva esta patología, las personas que participaron en las encuestas, tenían dificultades para cumplir con el tratamiento prescrito por el especialista, una de esas la concientización que sería la barrera principal para el cumplimiento de la misma, restando importancia al impacto que tiene sobre su salud el llevar el tratamiento bajo las directrices indicadas, por lo que se ve afectada la fidelidad de la misma ya sea en la forma farmacológica y en sus medidas en estrecha relación con los estilos de vida.

Palabras claves: hipertensión arterial, adherencia, terapéutica, farmacológico, no farmacológico.

\section{Introducción.}

La conducta de adherencia a los tratamientos es el indicative más importantes a evaluar en las personas con factores de riesgo para la salud cardiovascular, ya que la no adherencia implica índices de morbimortalidad, considerable y preocupantes, los mismo que pueden tener consecuencias en el aspecto, económico, social, , individual y familiar, llamando así la atención lograr la adherencia a los tratamientos, considerándose así un meta basado en el análisis de experiencias de los pacientes, permitiendo así identificar dificultades que ponen barreras en la adherencia.

Para lograr un adecuado control de la hipertensión arterial es importante la educación y fortalecer el conocimiento disciplinar del fenómeno de la adherencia a los tratamientos, con el fin de contribuir y evitar complicaciones.

Según el ministerio de salud pública del Ecuador, han realizado varios estudios sobre prevalencia de hipertensión arterial y la forma de comportamiento en las diferentes poblaciones y se ha establecido las causas y tratamiento de la misma, las cuales deben aplicarse según las características de las personas.

En el país se han implementado guías clínicas para el manejo integral de este problema de salud.

Con estos antecedentes, se realizará la investigación en la parroquia de Honorato Vásquez del cantón Cañar, con el objetivo de describir y analizar la adherencia terapéutica en pacientes con hipertensión arterial y determinar el factor y de acuerdo a la percepción de la sintomatología y de acuerdo a los resultados llegar hacer un análisis de resultados, planificar intervenciones como promoción de estilos de vida saludables y prevención de complicaciones. 


\section{Introducción al problema.}

La adherencia debe ser considerada como una conducta compleja que consta de una estrecha relación entre la conducta del individuo y el querer participar y por ende su comprensión del tratamiento, actuando como un equipo, tanto el profesional de la salud como el paciente basados en una buena comunicación donde se puede identificar los factores, que puede potenciar la adherencia al tratamiento.

La educación, el establecimiento de objetivos y el soporte que brinda la sociedad y su familia pueden ayudar al paciente a controlar la hipertensión arterial.

Con estos antecedentes se puede recalcar, que se hace necesario que la enfermería valore en las personas con hipertensión arterial los factores que están relacionados con la adherencia al tratamiento.

La hipertensión arterial es una condición médica caracterizada por un incremento continuo de las cifras de presión arterial por encima de 140/90 mmHg.

Según la organización mundial de la salud y que está afectando a cerca de mil millones de personas a nivel mundial.

Hoy se conoce que la hipertensión arterial del adulto comienza muchas veces desde la niñez. Estudios recientes Organización Mundial de la Salud, han puesto de manifiesto la incidencia cada vez mayor de hipertensión arterial en los niños, con una prevalencia actual del 3 a $5 \%$ y con tendencia al aumento.

La disminución de los ejercicios físicos, cambios desfavorables en el estilo de vida entre los que se incluyen la tendencia al consumo de alimentos procesados y en conservas, que contienen una elevada concentración de sal, las llamadas comidas chatarra o comidas rápidas, y el estrés.

El comportamiento de la tensión arterial puede variar teniendo en cuenta características como la edad, el sexo, el grupo racial, el peso y la talla.

\section{Importancia del problema.}

El aumento en la magnitud y en la gravedad de la hipertensión arterial ha sobrepasado todas las expectativas, donde el problema ha crecido con tal rapidez que se reconoce como una epidemia al representar la tercera causa de muerte.

Existen diversas razones por las que el análisis de este tipo de afecciones resulta importante. La primera es que representan el principal factor de riesgo de afecciones cardiovasculares siendo la segunda causa de muerte no violenta en hombres y mujeres. 
Se constituye en un problema de salud pública por su alta prevalencia de muertes., la hipertensión arterial, es un trastorno de carácter multicausal, lo cual determina la existencia de diferentes vías etiológicas y obliga a la evaluación de efectos interactivos de distintas variables sobre la presión arterial.

La identificación de los factores de riesgo constituye una tarea importante para su prevención, tratamiento y control.

Dentro de los factores que han sido investigados en relación con los trastornos hipertensivos se destaca los factores de riesgo como el tabaquismo, consumo de alcohol, inadecuada alimentación caracterizada por el excesivo consumo de grasas saturadas y alimentos altos en energía contribuyen al aumento de sobrepeso y obesidad que junto al sedentarismo conllevan al deterioro de la salud y consecuentemente a la aparición de enfermedades crónicodegenerativas, entre ellas la hipertensión arterial, propias de las ciudades modernas del siglo XXI.

Con frecuencia se observa a pacientes hipertensos que señalan que su hipertensión es emotiva, pues reconocen que generalmente los incrementos en su presión arterial se asocian a situaciones estresantes aun cuando se encuentran bajo tratamiento con fármacos hipotensores.

Por esto, es importante el reconocimiento de temas como este, presentan a largo plazo enfermedades que pueden afectar su sistema cardiovascular y la salud como aspecto general, con estos antecedentes la parroquia Honorato Vasquez del cantón Cañar no queda excluidas del padecimiento en sus habitantes y conjuntamente con sus estilos de vida.

\section{Metodología.}

Se utilizó cuantitativo descriptivo, transversal lo que permitió describir la adherencia al tratamiento en una muestra aleatoria de 50 pacientes diagnosticados de hipertension arterial. Siendo protagonistas de forma voluntaria con consentimiento informado, el instrumento a utilizar una escala tipo Likert basada en el protocolo propuesto por Colás y Buendía, guiado a: puntualizar la variable a medir que en la presente investigación corresponde al nivel de adherencia al tratamiento hipertensivo, formular los ítems o proposiciones acerca de la variable, seleccionar los ítems que más interesan eliminando los ambiguos, los que contienen una doble opinión y los que no miden lo mismo que los demás. La información se organizó en una base de datos en el programa Excel. 


\section{Resultados.}

Los participantes se caracterizaron por tener una edad mínima de 30 años y máxima de 90 años. La mayor frecuencia fue de > 50 años (100\%), sexo femenino (52\%), sexo masculino $(48 \%)$.

La mayor prevalencia de hipertensión arterial, se da en el sexo femenino. Los participantes presentaron bajo nivel educativo y socioeconómico, de los 50 pacientes tienen: primaria incompleta $64 \%$ y primaria completa $36 \%$, superior $0 \%$. El $66 \%$ se dedica a la agricultura y a la ganadería $34 \%$.

Instrumento, que se presenta a continuación, consta de 9 ítems con 3 alternativas de respuesta: totalmente de acuerdo, parcialmente de acuerdo, totalmente en desacuerdo, luego de firmado el consentimiento informado, se aplica de forma individual, con items que el paciente hipertenso conoce de forma básica.

Instrumento de conocimientos generales de tratamiento hipertensivo.

Estimado (a) paciente hipertenso: Con el objeto de fortalecerle su adherencia terapéutica, a continuación se presentan una serie de afirmaciones que debe contestar en concordancia a su criterio personal.

Por favor, marque con una $\mathrm{X}$ la respuesta que más se aproxime a su convicción. Convenciones: TA: Totalmente de acuerdo; PA: Parcialmente de acuerdo; TD: Totalmente en desacuerdo.

Tabla N1 Preguntas paciente hipertenso

\begin{tabular}{|c|c|c|c|c|}
\hline $\mathbf{N}^{\circ}$ & Ítem & TA & $\mathbf{P A}$ & TD \\
\hline 1 & Conoce las complicaciones que puede ocasionar la hipertensión arterial & 28 & 10 & 12 \\
\hline 2 & Cumple el tratamiento indicado por su medico & 17 & 24 & 9 \\
\hline 3 & $\begin{array}{l}\text { Asiste a las consultas programadas por su médico para el seguimiento de la hipertensión } \\
\text { arterial }\end{array}$ & 21 & 8 & 21 \\
\hline 4 & Cuando no cumple el tratamiento, se lo comunica a su médico y/o enfermera & 18 & 28 & 4 \\
\hline 5 & $\begin{array}{l}\text { Recibe de su médico y/o enfermera la información suficiente para comprender el } \\
\text { tratamiento }\end{array}$ & 16 & 15 & 19 \\
\hline 6 & $\begin{array}{l}\text { Recibe la educación por el médico y/o enfermera de forma individual y rara vez } \\
\text { colectiva }\end{array}$ & 18 & 21 & 11 \\
\hline 7 & Se siente beneficiado (a) con el tratamiento o recomendación indicado & 34 & 16 & 0 \\
\hline
\end{tabular}


8 Se siente vulnerable a las complicaciones de la hipertensión arterial

$27 \quad 18 \quad 5$

9 Siente la hipertensión arterial como una amenaza para su salud

$44 \quad 6 \quad 0$

Fuente: Autores de la investigación. Ecuador 2018.

Grafico $\mathbf{N}^{\circ} 1$ Instrumento de conocimiento generales de tratamiento hipertenso.

\section{Instrumento de conocimientos generales de tratamiento hipertensivo}

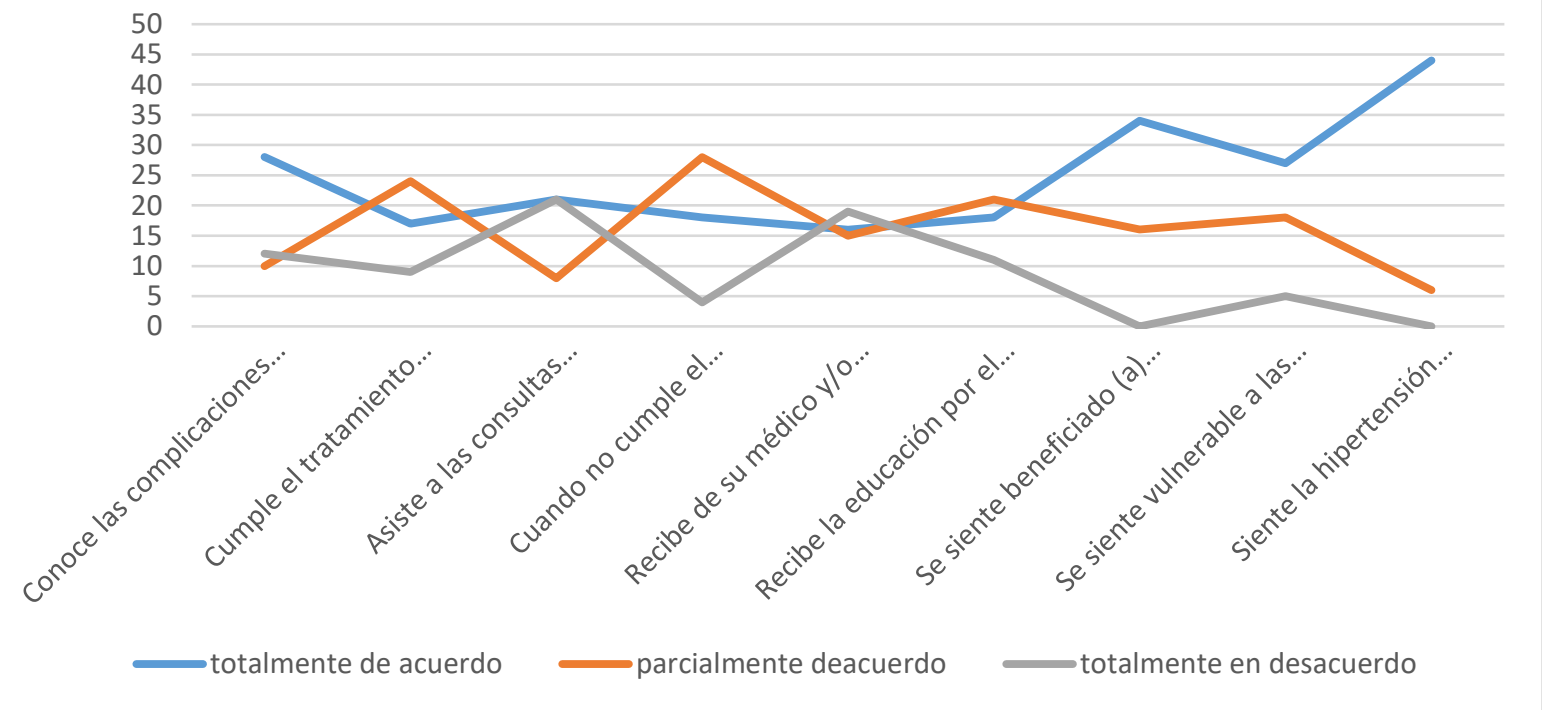

Fuente: Autores de la investigación. Ecuador 2018.

\section{Conclusiones.}

- Este estudio se encamina a analizar e identificar problemas reales y potenciales del paciente hipertenso y su adherencia al tratamiento, la búsqueda de favorecer y optimizer el tratamiento para esta patología, error que se dan por la falta de información, e interés por parte del paciente, sumado así la comunicación deficiente entre el equipo de salud y los pacientes, además buscar estrategias para mejorar de la 
salud de los individuos con el apoyo de su vincula social y familiar, la falta de percepción sobre la complicaciones que conlleva la enfermedad, situaciones que influyen de forma negativa en los objetivos del tratamiento.

- El instrumento utilizado ha sido probado en varios estudios resultando una herramienta adecuada, para la evaluación concreta y objetiva de forma cuantitativa la adherencia al tratamiento hipertensivo con enfoque de investigación educativa, comunicación e información, obtenidas en la literatura, hay que recalcar que los pacientes con deficiente adherencia al tratamiento podrían o no experimentar sintomatologia, y que podría tener consecuencias fatales por esta enfermedad bien llamada el asesino silencioso.

\section{Agradecimiento.}

Nuestro agradecimiento a las personas hipertensas de la comunidad de Honorato Vásquez que nos recibieron en sus hogares y nos otorgaron unos minutos de su tiempo

\section{Bibliográfia.}

- Colas, M. y Buendia, L. Investigación Educativa. Sevilla, España: Ediciones Alfar. 1998.

- Canales, M. Metodología de investigación social. Introducción al oficio. Chile: LOM Ediciones. 2006.

- Granados Gámez, G., Gil Roales-Nieto, J. e Ybarra Sagarduy, J.L. Un estudio exploratorio sobre el desarrollo de creencias sobre síntomas como señales de hipertensión arterial. Psicothe-ma. 2006; 18: 822-827.

- . Granados Gámez, G., Gil Roales-Nieto, J. e Ybarra Sagarduy, J.L. Un estudio exploratorio sobre el

- desarrollo de creencias sobre síntomas como señales de hipertensión arterial. Psicothema. 2006; 18 :

- 822-827.

- Higgins, L.J. The associations of personal body awareness, symptom perception, and time line anticipation

- with adherence behavior of adults with primary hypertension. Doctoral

- Burnier M. Conformidad con el tratamiento para la hipertensión arterial. EDTNA/ERCA J. 2005:157-61.

- Martín Alfonso L. Adherencia al tratamiento en hipertensos de áreas de salud del nivel primario.2003-2007

- [tesis]. La Habana: Escuela Nacional de Salud Pública; 2009 [citado 2 Sept 2014]. Disponible en: http://www.revsalud.sld.cu 
- Colas, M. y Buendia, L. Investigación Educativa. Sevilla, España: Ediciones Alfar. 1998.

- Canales, M. Metodología de investigación social. Introducción al oficio. Chile: LOM Ediciones. 2006.

- Granados Gámez, G., Gil Roales-Nieto, J. e Ybarra Sagarduy, J.L. Un estudio exploratorio sobre el

- desarrollo de creencias sobre síntomas como señales de hipertensión arterial. Psicothema. 2006; 18:

- 822-827. 
Para citar el artículo indexado.

Bustamante L., Cruz M. \& Aguaiza M... (2018). Adherencia terapéutica de pacientes hipertensos de la comunidad de Honorato Vasquez. Revista electrónica Ciencia Digital 2(3), 633-641. Recuperado desde: http://cienciadigital.org/revistacienciadigital2/index.php/CienciaDigital/article/view/180/16 $\underline{0}$

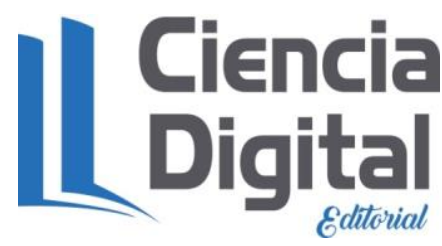

El artículo que se publica es de exclusiva responsabilidad de los autores y no necesariamente reflejan el pensamiento de la Revista Ciencia Digital.

El articulo queda en propiedad de la revista y, por tanto, su publicación parcial y/o total en otro medio tiene que ser autorizado por el director de la Revista Ciencia Digital.
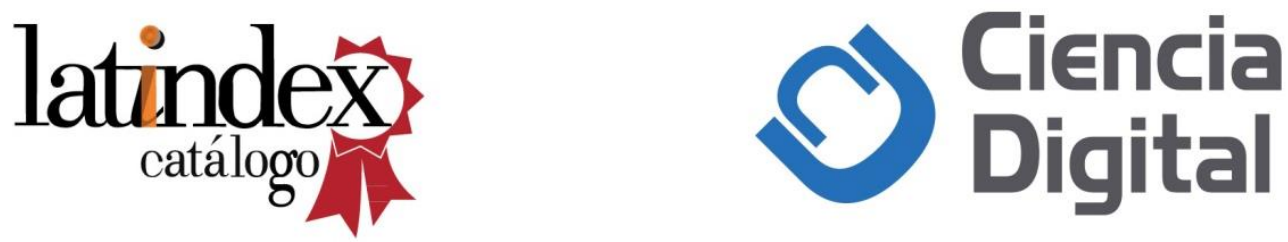